\section{$\underset{\text { hommes }}{\text { \& migrations }}$}

\section{Hommes \& migrations}

Revue française de référence sur les dynamiques

migratoires

$1315 \mid 2016$

Ondes de choc

\title{
Les djihadistes à travers Le Monde
}

Pluralité des analyses et impensés

\section{Carine Guérandel et Éric Marlière}

\section{OpenEdition \\ 1 Journals}

\section{Édition électronique}

URL : http://journals.openedition.org/hommesmigrations/3705

DOI : 10.4000/hommesmigrations.3705

ISSN : 2262-3353

\section{Éditeur}

Musée national de l'histoire de l'immigration

\section{Édition imprimée}

Date de publication : 1 juillet 2016

Pagination : 9-16

ISBN : 978-2-919040-36-0

ISSN : 1142-852X

\section{Référence électronique}

Carine Guérandel et Éric Marlière, «Les djihadistes à travers Le Monde», Hommes \& migrations [En ligne], 1315 | 2016, mis en ligne le 01 juillet 2019, consulté le 02 janvier 2020. URL : http://

journals.openedition.org/hommesmigrations/3705; DOI : 10.4000/hommesmigrations.3705 


\title{
LES DJIHADISTES \\ À TRAVERS LE MONDE PLURALITÉ DES ANALYSES ET IMPENSÉS
}

Par CARINE GUÉRANDEL, maître de conférences, et ÉRIC MARLIÈRE, maître de conférences, université de Lille (CeRIES - Centre de recherche "Individus Épreuves Sociétés »).

\author{
Suite aux attentats de 2015, la presse française se fait l'écho \\ de la parole des intellectuels afin de donner sens à cette violence \\ insensée. Dans les colonnes du quotidien Le Monde se croisent \\ des chercheurs de plusieurs disciplines et horizons idéologiques, \\ attelés ensemble à la lourde tâche de décrypter la genèse \\ du terrorisme djihadiste dans I'Hexagone. Face à des dynamiques \\ de radicalisation aux origines multiples, les modèles explicatifs \\ invoqués divergent. L'étude de leur mise en scène médiatique \\ permet d'entrer dans la fabrique des représentations collectives \\ des groupes sociaux et d'identifier les angles morts de l'analyse.
}

À la suite des travaux de Mathieu Rigouste ${ }^{1}$ sur les représentations dominantes des « cités » produites par les médias ou de Fabien Beyriaª sur le traitement médiatique du match de football FranceAlgérie du 6 octobre 2011, cet article vise à appréhender les liens établis entre la jeunesse populaire urbaine et la question terroriste dans les articles de presse publiés dans Le Monde suite aux attentats du 13 novembre 2015. Quotidien français de référence, considéré comme ayant une sensibilité de " centre gauche " même s'il se revendique non partisan ${ }^{3}$, essentiellement lu par les classes moyennes et supérieures mieux dotées a priori en capital scolaire et culturel, Le Monde se donne pour objectif de « raconter, décrire, expliquer l'onde de choc de ces attentats » à partir «d'un travail de suivi au long cours » et "en multipliant les angles, les formats, en variant les types de sujets, en creusant tous les aspects ${ }^{4} »$. Ainsi, au-delà du récit des faits, des portraits des kamikazes et de leurs victimes ou encore de la présentation des mesures politiques afférentes aux événements, le journal propose également des pages «Enquête » et « Débats » donnant la parole à des spécialistes d'horizons variés qui s'opposent ou se nuancent dans l'appréciation des causes du terrorisme. En cela, il constitue un analyseur privilégié des controverses et des oppositions relatives aux origines du djihadisme - 
relayées et, dans le même mouvement, produites par les médias - à l'œuvre dans le champ de la recherche et dans le monde politique.

Il s'agit d'interroger les ressorts et la nature des cadres d'intelligibilité des analyses produites par les " experts », ou du moins ceux admis comme tels par le journal. Nous avons fait le

Il s'agit d'interroger

les ressorts et la nature

des cadres d'intelligibilité

des analyses produites

par les " experts»,

ou du moins ceux admis

comme tels par le journal. jeunes en quête de sens. C'est notamment la thèse défendue par le politiste Olivier Roy qui évoque "l'islamisation de la radicalité ${ }^{8} »$. Selon lui, les jeunes motivés à faire le djihad, qu'ils soient de "la deuxième génération " ou des "convertis ", rejettent la culture et la religion de leurs parents. Ils adhèrent à un «islam de rupture », ancré dans un « individualisme forcené ». Pour les premiers, la culture occidentale à laquelle ils appartiennent (consommation de cannabis, d'alcool, fréquentation des boîtes de nuit, délinquance) devient le "symbole de leur haine de soi ». L'islam leur permet alors de reprendre "à leur compte une identité que leurs parents ont, à leurs yeux, galvaudée ». Les seconds "choisissent l'islam parce qu'il n'y a que ça sur le marché de la révolte radicale (...). Rejoindre Daech, c'est la certitude de terroriser ». L'auteur insiste ainsi sur leur position de rupture tant avec leur famille (qui ne comprend pas leur engagement) qu'avec les communautés musulmanes (ni études religieuses sérieuses, ni pratiques communautaires comme le ramadan avant leur radicalisation) qui passe notamment par la constitution d'une nouvelle fraternité centrée sur un petit groupe de copains. Comme le souligne Rik Coolsaet, spécialiste des relations internationales, les jeunes combattants belges partis en Syrie sont des « radicaux islamisés » en "quelques semaines tout au plus » qui «connaissent mieux les techniques de vol de voiture que les sourates $d u \operatorname{Coran}^{9}$ ». Le psychanalyste Serge Hefez valide également la thèse de l'islamisation de la "révolte adolescente » en la considérant comme une crise "radicale» qui "transforme le foyer familial en champ de bataille ${ }^{10}$ ». Selon lui, ceux qui tombent dans le piège de la radicalisation loin d'être des marginaux (familles de la classe moyenne laïque, catholique, juive ou musulmane peu pratiquante) - ressemblent aux jeunes filles anorexiques, aux adolescents toxicomanes ou à ceux qui se scarifient. Dans tous les cas, il s'agit de

Un premier courant d'idée d'inspiration philosophique et psychologique considère que l'engagement dans le djihadisme relève avant tout d'une révolte moderne nihiliste et générationnelle de

\section{Djihadistes en rupture : entre révolte violente nihiliste et orgueil}

\footnotetext{
5. Le corpus de source qui a fait l'objet d'une analyse de contenu qualitative se compose de l'ensemble des articles publiés entre le 14 novembre 2015 et le 31 mars 2016 sur la thématique. Aussi, les données renvoient à tous les papiers, chroniques ou interviews des " experts »-chercheurs en sciences sociales, intellectuels médiatiques, juristes, écrivains, journalistes spécialisés - qui se donnent comme objectifs d'expliquer les origines du terrorisme. 6 . Aucun théoricien n'a été écarté de l'analyse. Si certains ne figurent pas dans cet article - comme par exemple, le politologue François Burgat considéré comme un spécialiste de l'islamisme (voir Chahla Chafiq, Islam politique, sexe et genre. À la lumière de l'expérience iranienne, Paris, PUF, 2011) -, c'est parce qu'ils ne prennent pas la parole (ou que le journal ne la leur donne pas) au cours de la période étudiée. 7. Patrick Champagne, Faire l'opinion. Le nouveau jeu politique, Paris, Minuit, 1990. 8. Le Monde, 25/11/2015, p. 14. 9. Le Monde, 27-28/03/2016, p. 25. 10. Le Monde, 15/12/2015, p. 24.
} 
" mettre leur mort en jeu pour tenter de donner du sens à leur existence ». Leur rencontre avec un rabatteur qui les valorise narcissiquement marque le début de «l'engrenage infernal». Les membres du groupe virtuel deviennent progressivement les seules personnes dignes de confiance. L'anthropologue Dounia Bouzar parle en ce sens d'« embrigadement relationnel » : le jeune se fait " absorber » par son nouveau groupe fusionnel "qui se présente comme une famille de substitution ${ }^{11}$ ".

À cela s'ajoute un " embrigadement cognitif » qui débute par une mise à disposition d'idéaux adaptés au profil psychologique du jeune et qui aboutit à "sa déshumanisation (...) et celle de ses futurs victimes ». Le psychanalyste Roland Gori valide ce double processus ${ }^{12}$. Selon lui, le terrorisme comme le mouvement fasciste se construit à la fois sur une fraternité fondée sur "le meurtre et le sang ", sur "l'effacement de la pitié et l'éloge de la cruauté ». Il s'agit d'" effacer ce qui constitue le socle de l'identification à l'humanité, à savoir la compassion". On retrouve cette idée de "dessèchement de lêtre » et d'individu "déshumanisé " dans la tribune de l'écrivain Julien Suaudeau ${ }^{13}$. Selon lui, le terroriste, comme le tueur de masse, se comporte comme un «zombie ", " un fantôme » insensible et calme, " étranger aux émotions qui faisaient de lui un être humain ". Mais "peu importent leurs conditions sociales, leurs croyances, leurs illusions historiques ou politiques », les «djihadistes made in France ne sont rien d'autres que des loosers sans frontières et des citoyens du vide ". Il s'agit donc d'une "violence moderne " associée à " une volonté de revanche sur une frustration rentrée ${ }^{14}$ ».

Roy récuse l'idée d'avoir affaire à de jeunes utopistes - puisqu'ils ne s'intéresseraient pas vraiment à la société civile lorsqu'ils sont en Syrie et qu'ils s'attribueraient des esclaves sexuelles - et préfère parler de "nihilisme générationnel » qu'il associe à la logique de l'orgueil retrouvé. Comme le défend également le politiste Xavier Crettiez ${ }^{15}$, la violence des attentats parisiens est moins lisible que celle que pouvaient porter certains révolutionnaires ou nationalistes cherchant à "faire fuir "l'occupant colonial" ", renverser "l'État bourgeois ", libérer "des patriotes emprisonnés». Elle renvoie ici au « délire de toute-puissance ", à la "volonté de reconnaissance » : "lobjectif semblait être de tuer et uniquement de tuer!" Il en conclut qu' «à un terrorisme politique et idéologique s'est substitué un terrorisme apocalyptique d'inspiration théologique ».

Ces auteurs se focalisent avant tout sur les failles identitaires
Ces auteurs se focalisent avant tout sur les failles identitaires des jeunes terroristes et considèrent l'engagement djihadiste comme la conséquence d'une révolte nihiliste adolescente ou plus largement générationnelle. des jeunes terroristes et considèrent l'engagement djihadiste comme la conséquence d'une révolte nihiliste adolescente ou plus largement générationnelle. Si ces auteurs ont le méritent d'éviter de penser la jeunesse de manière manichéenne avec, d'un côté, des " jeunes de cités » violents et ethnicisés et, de l'autre, les « bons jeunes ", intégrés et laïques, ils ont tendance, en revanche, à mettre la focale sur les ressorts psychologiques de l'action individuelle et à nier les logiques sociales. Ces approches passent, en effet, sous silence les processus sociaux qui amènent certains Français à adhérer aux discours de la radicalité et à devenir des meurtriers kamikazes prêts à détruire par des procédés extrêmes le monde dans lequel ils évoluent. Le terrorisme ne serait rien d'autre qu'un virus qui se propage dans la tête de jeunes révoltés ou en " crise », en quête de sens ou de vengeance narcissique désespérée, finissant par être totalement décérébré et déshumanisés par l'appareil idéologique. Comme ceux qui adhèrent à une secte, ces jeunes en rupture se tournent vers le djihadisme - qui « induit une régression de la pensée »- mais «leur radicalisation, en ce qu'elle témoigne d'un porte-à-faux avec le monde, aurait pu se résoudre autrement à travers une rencontre, une pratique artistique leur donnant une reconnaissance, la toxicomanie ou une autre forme d'addiction ${ }^{16}$ ". La religion 
est alors interprétée en termes de "plein contre vide », d'appartenance, d'engagement, d'identité dans un monde perçu comme suspect, menaçant, polluant, matérialiste et hostile ${ }^{17}$. Autrement dit, David Le Breton nous explique que l'acte terroriste aurait pu se concrétiser d'une manière différente et ses jeunes se seraient " exprimés » dans d'autres domaines. La thèse nihiliste développée rapidement ici s'oppose donc aux lectures culturalistes qui voient dans l'islam les sources du mal et qui prônent une radicalisation de la population musulmane.

\section{Djihadistes fondamentalistes : la rhétorique du choc des civilisations}

Des spécialistes de l'islam et du monde arabe considèrent que l'analyse "nihiliste » néglige la dimension religieuse du terrorisme. Les porteurs des thèses culturalistes préfèrent ainsi rechercher les explications de la violence dans la religion et plus généralement dans la

Si la religion apparaît comme

un objet d'étude tout à fait

intéressant pour comprendre le monde social, les défenseurs des thèses culturalistes tendent

à essentialiser les cultures

et les identités en cherchant

dans l'essence de l'islam, et

notamment dans sa version

radicale, les raisons profondes

de la violence terroriste. « radicalisation de l'islam ${ }^{20}$ ». Les terroristes «salafisés » veulent "abattre la civilisation européenne et ses valeurs, grâce à cette rupture culturelle fondamentale prônée par le salafisme ". Il précise que "même dans sa version non-djihadiste, le salafisme fait sécession avec la République et les "mécréants" dont "le sang est licite" ». D'après l'historien belge Pierre Vermeren, l'indifférence des classes politiques belge et française aux questions religieuses laisse en effet le champ libre aux prédicateurs salafistes notamment Iraniens et Saoudiens - qui œuvrent à " la radicalisation de la jeunesse musulmane dans le monde ${ }^{21}$ » en jouant sur leur culpabilité. Dans la même perspective, le sociologue Hugues Lagrange décrit les terroristes du 13 novembre comme des jeunes de cités en échec scolaire qui « ont nourri au fil des ans une haine de l'Occident. Les marginaux sont devenus des salafistes, les salafistes des djihadistes, les djihadistes des kamikazes d'Allah. L'islam ne s'est pas emparé d'une radicalité disponible (...). Culture et religiosité sont complètement mêlées ${ }^{22}$ ". Il ne conçoit donc pas d'analyse du terrorisme sans référence " aux migrations du Sud dans une Europe chancelante ", "à la dérive de l'islam et aux guerres civiles qui ensanglantent les sociétés du Moyen-Orient ». Adhérer à "l'islam radical », c'est en effet " une "dignité" retrouvée, un mode de vie alternatif à celui de la modernité occidentale et un sens, par le sacrifice de soi et le meurtre d'autrui ».

L'historien Marcel Gauchet confirme que la violence terroriste est bien "un phénomène religieux $x^{23}$ ». Cependant, il considère que la résurgence du fondamentalisme islamique, "symbolisée par le retour de la charia », témoigne paradoxalement de la «sortie du religieux » provoquée par la mondialisation ou «l'occidentalisation culturelle du globe ». S'il précise que le fondamentalisme peut concerner l'ensemble des religions, il existe selon lui « une forte spécificité et une virulence particulière du fondamentalisme islamique » liée "à une conflictualité spécifique de la relation entre l'islam et les religions occidentales". Ainsi, le message fondamentaliste "entre en résonance avec les difficultés de l'acculturation " de la " jeunesse immigrée » (sous-entendu les jeunes des banlieues) « à une culture individualiste en rupture totale avec ses repères, y compris communautaires, qui viennent de sa tradition religieuse ». Lors des attentats du 13 novembre, «le choix des cibles est très 
peu politique » : ils ont tiré sur «ce à quoi ils aspirent tout en le refusant radicalement. Ils se détruisent de ne pas pouvoir assumer le désir qu'ils en ont ».

Dans sa chronique, Alain Frachon explique que "l'Occident doit être attaqué parce qu'il est responsable de la décadence morale de lislam. Paris, ville des plaisirs, n'est-ce pas, Paris doit payer ${ }^{24}$ ». Pour résumer, les jeunes radicalisés adhèrent à une vision du monde "apocalyptique, manichéenne, de fin de l'Occident et de triomphe de l'Orient ${ }^{25}$ ". Comme le rappelle Kepel, Daech menace les Occidentaux et particulièrement les "sales et méchants Français » depuis 2014 ${ }^{26}$. Les attentats de janvier 2015 visaient les « islamophobes » à travers la rédaction de Charlie, les " apostats " avec "les policiers musulmans qui servaient sous l'uniforme français » et les juifs lors de la prise d'otage de l'Hyper Cacher. Mais ceux de novembre ciblent tous les citoyens français dans l'espoir de "déclencher une guerre civile » et de "faire vaciller la République » en mettant « à feu et à sang l'Europe, perçu comme le ventre mou de l'Occident " pour reprendre les propos d'Abou Moussab Al-Souri dans son Appel à la résistance islamique mondiale ${ }^{27}$. Ainsi, si Gilles Kepel refuse le "tabou de l'islamophobie ", il en va de même de certaines personnalités de gauche, dont des intellectuels comme Élisabeth Badinter ou Marcel Gauchet qui «se mobilisent pour la laïcité» dans un souci intégrationniste, en dénonçant "le déni pratiqué par les islamo-gauchiste à l'égard de la montée en puissance de l'islamisme radical » tout en disant rejeter «le racisme arabo-musulman ${ }^{28}$ ».

Si la religion apparaît comme un objet d'étude tout à fait intéressant pour comprendre le monde social, les défenseurs des thèses culturalistes tendent à essentialiser les cultures et les identités en cherchant dans l'essence de l'islam, et notamment dans sa version radicale, les raisons profondes de la violence terroriste. Selon eux, la culture musulmane semble incompatible avec la civilisation occidentale et, plus encore, avec la république française qui a inscrit la laïcité dans sa constitution. De fait, en opposant «le social » au "religieux », en réactivant la rhétorique du "choc des civilisations ", en considérant la radicalisation de l'islam comme une «maladie » qui arriverait du Moyen-Orient et se propagerait sur le sol européen, ces explications évacuent la dimension historique et sociale de la religion envisagée comme un système de pensées et de croyances. Or, comme le rappelle Bernard Lahire, ce n'est pas parce que "les acteurs se revendiquent de l'islam » que " c'est uniquement l'islam qui est en cause ${ }^{29}$ ». Fina-
Ces analyses du terrorisme mettent l'accent sur l'instabilité du monde musulman héritée de la colonisation et des conflits internationaux récents. Pour ces auteurs, il existe une homologie entre les injustices subies par les populations musulmanes et le désir de revanche d'une certaine jeunesse européenne frustrée, déclassée ou prise dans l'ennui. nant sur la compatibilité de l'islam avec les valeurs démocratiques, les discours culturalistes, qui réactivent une vision menaçante et obscurantiste de la religion, participent à alimenter la stigmatisation de l'ensemble de la communauté musulmane, première victime du terrorisme.

\section{Djihadistes globalisés : entre problématique postcoloniale et expérience de la relégation sociale}

Si les analyses nihiliste et culturaliste s'opposent sur un certain nombre de points étudiés à l'instant, elles font en quelque sorte front commun contre ce qu'elles nomment comme les thèses " tiers-mondialistes ", relatives à " la souffrance postcoloniale, l'identification des jeunes à la cause palestinienne, leur rejet des interventions occidentales au Moyen-Orient et leur sentiment d'exclusion d'une France raciste et islamophobe "(Olivier Roy ${ }^{30}$ ), puisque le phénomène ne touche qu'une minorité de la jeunesse aux caractéristiques sociologiques variées. Les facteurs économiques et sociaux joueraient "tout au plus un rôle de déclencheur" 
(Marcel Gauchet ${ }^{31}$ ). Néanmoins, des chercheurs expliquent le terrorisme perpétré par de jeunes français en se référant à des causes structurelles telles que la colonisation, les conflits internationaux et le déficit d'intégration de certains jeunes européens.

L'historien Matthieu Rey analyse l'émergence de l'État islamique (EI) en relation avec la guerre irakienne et la révolution syrienne qui ont pour matrice commune trente années de règne du parti Baas (laïque, autoritaire et nationaliste). Par le biais des réseaux sociaux, Il semblerait, en effet, l'EI profite, selon lui, de la que l'on passe d'un conflit "situation régionale moyen-

declasse initiépar une orientale devenue totaleminorité « active » politisée et révolutionnaire dans les pays développés à une hostilité Nord/Sud - inscrite dans des rapports inégalitaires

- matérialisée par un

mécontentement populaire de masse grandissant et de plus en plus virulent à

l'égard de l’occident. ment injuste pour la majorité » afin de se positionner comme le défenseur des opprimés "face à un Occident qui laisse faire ${ }^{32}$ ". Dans le même temps, il devient "l'agent à même de transformer une dénonciation locale, en Europe, des jeunes en mal de sens, en une quête humanitaire qu'il traduit dans une série d'action d'une extrême violence ». De manière très proche, on peut lire dans la recension du dernier ouvrage de Raphaël Lioger, La Guerre des civilisations n'aura pas lieu. Coexistence et violence au XX $X^{\text {esiecles3, }}$ que le djihad relève avant tout de la colonisation de l'Afrique et du ProcheOrient articulée au développement des technologies de communication, à l'instabilité du monde et au désir de vengeance ${ }^{34}$. Comme le précise l'historien Jean-Pierre Filiu, l'essor du recrutement djihadiste se situe dans « une dynamique européenne » en trois phases : le recul de l'administration Obama au moment de sanctionner le régime de Bachar al-Assad pour le bombardement chimique de sa propre population en août 2013 ; les raids aériens contre l'EI en Irak puis en Syrie durant l'été 2014 dans le cadre d'une coalition menée par les ÉtatsUnis ; le soutien de Vladimir Poutine (avec la caution de l'Église orthodoxe) au dictateur Syrien par des bombardements concentrés sur les forces révolutionnaires ce même automne ${ }^{35}$. Aussi, pour reprendre l'analyse du politologue Bernard Rougier, on passe d'un «djihadisme stratégique » (avant la guerre d'Afghanistan) à un "djihadisme social » (après l'occupation de l'Irak), c'est-à-dire " un djihad par le "bas", fondé sur la pratique et la diffusion de l'ultraviolence comme instruments de promotion des sans-grade ", les prolétaires sunnites mais aussi les jeunes « délinquants déscolarisés » d'Europe ${ }^{36}$. Partir en Orient leur permet « d'échapper à la culpabilité de l'échec social en s'inventant un destin grandiose de "soldat de l'islam", commettre en retour des attentats en Europe apparait pour eux comme un moyen de changer de statut au sein de l'organisation EI en échappant à l'accomplissement des tâches subalternes comme l'inhumation des cadavres de "mécréants" et d" apostats" syriens $^{37}$ ».

Pour la politiste Delphine Placidi-Frot, l'ampleur, la visibilité et la médiatisation "en temps réel " des "inégalités sociales et économiques à l'échelle internationales et au sein des États" permet donc aux «entrepreneurs de violence » de mobiliser des individus frustrés ${ }^{38}$. Si le philosophe allemand Jürgen Habermas rappelle les processus historiques qui ont abouti à la déstabilisation du ProcheOrient, il attire l'attention sur « les destins ratés de l'intégration dans les foyers sociaux de nos grandes villes $^{39}$ ». Farhad Khosrokhavar explique, en ce sens, que les auteurs des attentats sont des jeunes " déclassés des cités » animés par une haine contre l'Europe "qui les a vus naître et les a plus ou moins mal éduqués ${ }^{40}$ ". Mais, en plus des jeunes " "désaffiliés", victimes de marginalisation ", de "stigmatisation " et d'" islamophobie " qui montrent en retour "un atavisme antifrançais en lien avec leurs origines (...) marocaine et algérienne ", l'auteur note l'essor des jeunes des classes moyennes "aux ressources intellectuelles et culturelles plus importantes», 
des convertis qui cherchent soit à "se venger de leurs conditions sociales désavantageuses (le cas des "petits blancs" qui agissent comme les jeunes des banlieues) », soit à faire de "l'humanitaire engagé $e^{41}$ ». Il existe donc deux types de djihadistes : " ceux qui souffrent » et "ceux qui s'ennuient » en quête "d'une existence festive ».

Ces analyses du terrorisme mettent l'accent sur l'instabilité du monde musulman héritée de la colonisation et des conflits internationaux récents. Pour ces auteurs, il existe une homologie entre les injustices subies par les populations musulmanes et le désir de revanche d'une certaine jeunesse européenne frustrée, déclassée ou prise dans l'ennui ${ }^{42}$. Les historiens Sophie Bessis et Mohamed Harbi dénoncent également la politique internationale des Occidentaux qui, d'une part, «se refusent à faire appliquer les résolutions de l'ONU susceptibles de résoudre » le conflit israélo-palestinien, et qui, d'autre part, "addiction au pétrole aidant ", soutiennent de "sanglants dictateurs » et des États participant à l'essor de "l'extrémisme islamiste ${ }^{43}$ ». Dans le même temps, les deux auteurs concèdent que les racines du mal terroriste sont nombreuses et multifactorielles et citent, entre autres, «l'abandon des banlieues » ou l'école. Mais ces dimensions ne sont jamais réellement abordées au travers d'une analyse fine de parcours de vie de jeunes et restent cantonnées à l'évocation très générale de déterminismes macrosociologiques.

\section{Conclusion}

Il semble difficile de conclure un tel travail tant le sujet reste polémique et les interprétations relativement éclectiques selon les domaines de recherche ou d'expertise des auteurs. Les analystes nihilistes avec un versant psychologisant tendent à déshumaniser le djihadiste ou le jeune radicalisé présenté comme décérébré et barbare. Les thèses autour de la violence de l'islam en appellent davantage au conflit de civilisation et appréhendent la religion comme une ressource pathologique et une valeur nocive opposée à l'Occident à laquelle se réfèrent des jeunes en rupture avec la société française. Enfin, un troisième courant, moins visible, se penche sur les conséquences historiques de la colonisation et, plus près de nous, de la guerre du Golfe, en évoquant le sentiment de revanche d'une jeunesse socialement frustrée et en défaut d'intégration. Si la variété des explications permet d'éviter toute uniformisation caricaturale de l'interprétation du phénomène, on note cependant certains angles morts : les ressorts sociaux de l'engagement religieux et guerrier ainsi que l'impensé des motivations politiques.

Alors que le phénomène du terrorisme international n'est pas nouveau ${ }^{44}$, on remarque l'absence d'analogie avec les expériences passées, notamment celles intrinsèques à l'extrême gauche utilisant la violence politique dans les années $1970^{45}$. Sur certains points, les idéologies et méthodes maoïstes se révèlent pourtant comparables à celles mises en place par Daech, notamment dans leurs orientations totalitaires et extrêmes ${ }^{46}$. Dans le même temps, il paraît essentiel de s'interroger sur les déplacements des antagonismes sociaux en l'espace de trente ans pour mieux saisir le phénomène actuel. Il semblerait, en effet, que l'on passe d'un conflit de classe initié par une minorité " active » politisée et révolutionnaire dans les pays développés ${ }^{47}$ à une hostilité Nord/Sud - inscrite dans des rapports inégalitaires - matérialisée par un mécontentement populaire de masse grandissant et de plus en plus virulent à l'égard de l'Occident $^{48}$. Sur le plan national, les contraintes économiques et sociales, qui prennent corps dans

41. Le Monde, 25/03/2016, p. 24. 42. Jean-Pierre Filiu, Les Arabes, leur destin et le nôtre. Histoire d'une libération, Paris, La Découverte, 2015 ; Farhad Khosrokhavar, La radicalisation, Paris, éd. de la MSH, 2014. 43. Le Monde, 18/11/2015, p. 23.

44. Il en est même consubstantiel et la globalisation en marquerait un nouvel âge avec l'accélération des échanges et des communications. Voir Jenny Raflik, Terrorisme et mondialisation. Approches historiques, Paris, Gallimard, 2016.

45. Isabelle Sommier, La violence révolutionnaire, op. cit. 46. Marc Lemaire, Dans le piège de la guerre insurrectionnelle. L'Occident à l'épreuve du communisme hier, de l'islamisme aujourd'hui, Paris, L'Harmattan, 2016. 47. Isabelle Sommier, La violence politique et son deuil. L'après 68 en France et en Italie, Rennes, PUR, 1998. 48. Arjun Appadurai, Géographie de la colère. La violence à I'usage de la globalisation, Paris, Payot \& Rivage, 2009. 
les habitus structurant les représentations et les actions des agents, ne sont pas suffisamment étayées au niveau de l'appréhension de la violence symbolique et des rapports de domination ${ }^{49}$, alors qu'elle affecte de manière spécifique les trajectoires sociales des jeunes des "quartiers ", générant souvent de la souffrance et de l'amertume chez beaucoup d'entre eux ${ }^{50}$. D'ailleurs, le déficit de références aux travaux ethnographiques traitant des quartiers populaires urbains questionne fortement puisque ces derniers développent, chacun à leur façon, les registres de la ségrégation urbaine, des déterminismes sociaux et des processus de discrimination structurant depuis trente ans la vie dans les cités ${ }^{51}$. Ainsi, aucun article ne présente d'enquête centrée sur les processus de socialisation - au sein des familles, entre pairs, dans le quartier ou le village, mais aussi médiatique et religieuse qui permettrait de cerner la particularité des parcours de vie, les points d'inflexion, de ruptures, de bifurcation, bref les conditions sociales de l'engagement violent. À quoi correspondent les modalités d'entrée dans la carrière de djihadistes ? Qu'en est-il des modes de socialisation institutionnelle qui parachèvent le processus dont le départ en Syrie paraît un élément déterminant ? Nous disposons de très peu d'éléments ici. Enfin, le dernier écueil est celui du politique. Les djihadistes seraient dépourvus de motivations politiques.
Pourtant, depuis les émeutes de 2005, des sociologues font la démonstration des significations politiques de certaines formes de violence comme, par exemple, le fait de brûler des bibliothèques dans un quartier populaire urbain ${ }^{52}$. Les tensions de plus en plus difficiles recensées depuis les années 1980 dans les «banlieues » traduisent, en effet, l'échec de la Marche pour l'égalité ${ }^{53}$, les diverses récupérations politiques ${ }^{54}$, la montée progressive du fait émeutier ${ }^{55}$ et le développement d'un sentiment d'injustice ${ }^{56}$ dont le djihadisme pourrait être un des aboutissements ${ }^{57}$. De manière originale, la sociologue Amel Boubekeur montre que la «perte d'identification à l'État » des jeunes français musulmans fascinés par Daech et «l'incapacité des élites à favoriser chez eux une participation citoyenne et critique » explique « la radicalisation de leur politisation »: Daech leur permet «de passer du statut d'esclaves de "l'impérialisme occidental" à celui d'adversaires politiques, enfin considérés par leurs États et les médias grâce à la peur qu'ils inspirent ${ }^{58}$ ".

Au final, le travail de recensement du Monde révèle le silence relatif des sociologues. L'une des explications se trouve peut-être dans le format médiatique qui contraint à expliquer des processus complexes en peu de lignes, de manière accessible et sans donner l'impression d'excuser les enquêtés comme l'affirment de manière simpliste les discours politiques sur les «excuses sociologiques ${ }^{59}$ ».

49. Pierre Bourdieu, Loïc Wacquant, Invitation à la sociologie réflexive, Paris, Seuil, 2014. 50. Stéphane Beaud, Michel Pialoux, Violences urbaines, violence sociale. Genèse des nouvelles classes dangereuses, Paris, Fayard, 2003.

51. Éric Marlière, «Les recompositions culturelles chez les “jeunes de cité” à l'épreuve des déterminismes sociaux et des effets du chômage, de la discrimination et de la ségrégation urbaine ", in Lien social et politique, n 70, 2013, pp. 103-117. 52. Denis Merklen, Pourquoi brûle-t-on des bibliothèques?, Paris, Presses de l'Enssib, 2013. 53. Abdellali Hajjat, La Marche pour l'égalité et contre le racisme, Paris, éd. Amsterdam, 2013. 54. Olivier Masclet, La Gauche et les cités. Enquête sur un rendez-vous manqué, Paris, La Dispute, 2003. 55. Stéphane Beaud, Olivier Masclet, "Des “marcheurs" de 1983 aux “émeutiers" de 2005 ", in Annales. Histoire, science sociale, $n^{\circ}$ 4, 2006. 56. Éric Marlière, La France nous a lâchés! Le sentiment d'injustice chez les jeunes de cité, Paris, Fayard, 2008. 57. Alain Bertho, Les enfants du chaos. Essai sur le temps des martyrs, Paris, La Découverte, 2016. 58. Le Monde, 26/01/2016, p. 13. 59. Bernard Lahire, Pour la sociologie. Et pour en finir avec une prétendue "culture de l'excuse ", Paris, La Découverte, 2016. 